\title{
Homoclinic Orbits of the FitzHugh-Nagumo Equation: Bifurcations in the Full System
}

\author{
John Guckenheimer* Christian Kuehn ${ }^{\dagger}$
}

\begin{abstract}
This paper investigates travelling wave solutions of the FitzHugh-Nagumo equation from the viewpoint of fast-slow dynamical systems. These solutions are homoclinic orbits of a three dimensional vector field depending upon system parameters of the FitzHugh-Nagumo model and the wave speed. Champneys et al. [A.R. Champneys, V. Kirk, E. Knobloch, B.E. Oldeman, and J. Sneyd, When Shilnikov meets Hopf in excitable systems, SIAM Journal of Applied Dynamical Systems, 6(4), 2007] observed sharp turns in the curves of homoclinic bifurcations in a two dimensional parameter space. This paper demonstrates numerically that these turns are located close to the intersection of two curves in the parameter space that locate non-transversal intersections of invariant manifolds of the three dimensional vector field. The relevant invariant manifolds in phase space are visualized. A geometrical model inspired by the numerical studies displays the sharp turns of the homoclinic bifurcations curves and yields quantitative predictions about multi-pulse and homoclinic orbits and periodic orbits that have not been resolved in the FitzHugh-Nagumo model. Further observations address the existence of canard explosions and mixed-mode oscillations.
\end{abstract}

\section{Introduction}

This paper investigates the three dimensional FitzHugh-Nagumo vector field defined by:

$$
\begin{aligned}
\epsilon \dot{x}_{1} & =x_{2} \\
\epsilon \dot{x}_{2} & =\frac{1}{\Delta}\left(s x_{2}-x_{1}\left(x_{1}-1\right)\left(\alpha-x_{1}\right)+y-p\right)=: \frac{1}{\Delta}\left(s x_{2}-f\left(x_{1}\right)+y-p\right) \\
\dot{y} & =\frac{1}{s}\left(x_{1}-y\right)
\end{aligned}
$$

where $p, s, \Delta, \alpha$ and $\epsilon$ are parameters. Our analysis views equations (1) as a fast-slow system with two fast variables and one slow variable. The dynamics of system (1) were studied extensively by Champneys et al. 4] with an emphasis on homoclinic orbits that represent travelling wave profiles of a partial differential equation [1]. Champneys et al. 4] used numerical continuation implemented in AUTO [7] to analyze the bifurcations of (1) for $\epsilon=0.01$ with varying $p$ and $s$. As in their studies, we choose $\Delta=5, \alpha=1 / 10$ for the numerical investigations in this paper. The main structure of the bifurcation diagram is shown in Figure 1.

Figure 1 shows a curve of Hopf bifurcations which is U-shaped and a curve of Shilnikov homoclinic bifurcations which is $\mathrm{C}$-shaped. Champneys et al. 4 observed that the $\mathrm{C}$-curve is a closed curve which folds back onto itself before it reaches the U-curve, and they discussed bifurcations that can "terminate" a curve of homoclinic bifurcations. Their analysis does not take into account the multiple-time scales of the vector field (1). This paper demonstrates that fast-slow analysis of the homoclinic curve yields deeper insight into the events that occur at the sharp turn of the homoclinic curve. We shall focus on the turning point at the top end of the C-curve and denote this region by $I$.

\footnotetext{
* Mathematics Department, Cornell University

${ }^{\dagger}$ Center for Applied Mathematics, Cornell University
} 


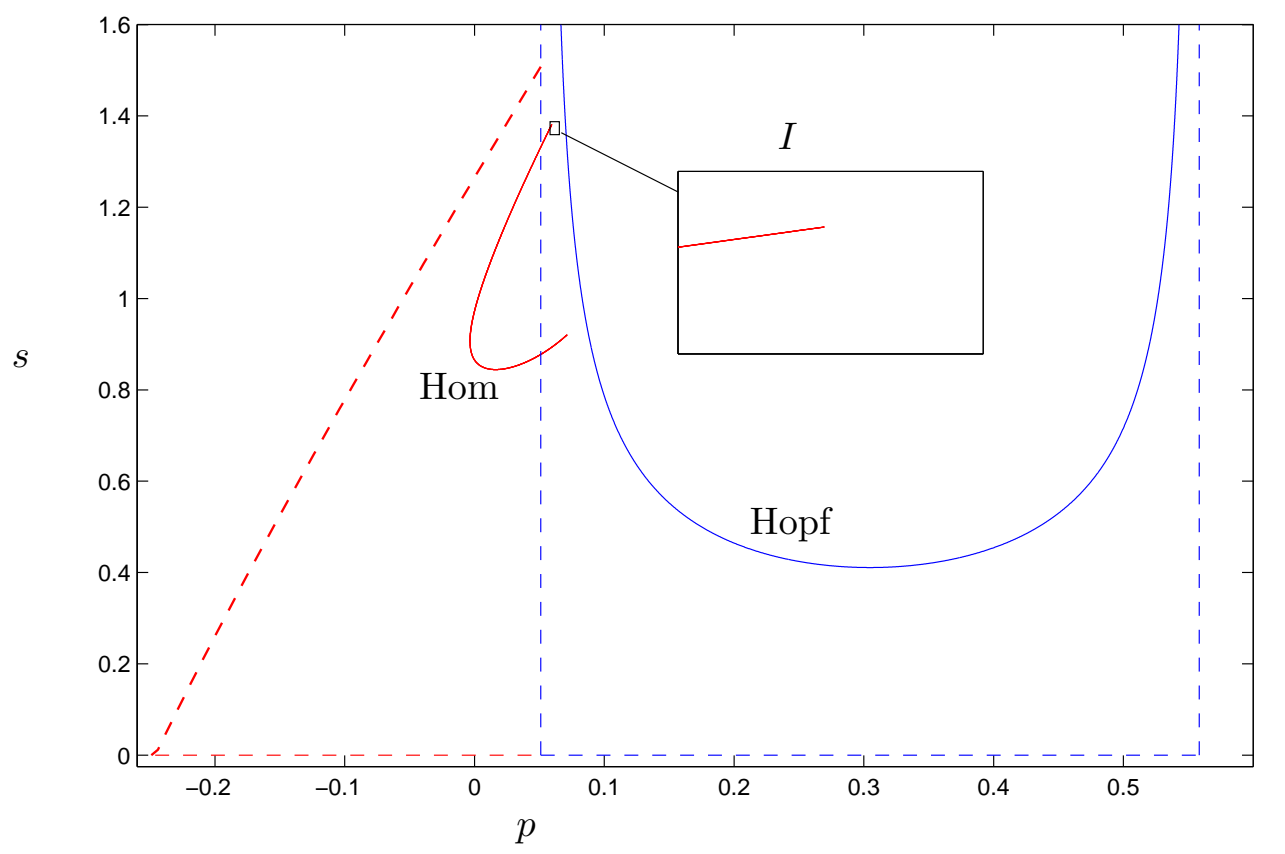

Figure 1: Bifurcation diagram for the FitzHugh-Nagumo equation (1). Shilnikov homoclinic bifurcations (solid red) and Hopf bifurcations (solid blue) are shown for $\epsilon=0.01$. The dashed curves show the singular limit $(\epsilon=0)$ bifurcation curves for the homoclinic and Hopf bifurcations; see [15] and Section 2 for details on the singular limit part of the diagram.

We regard $\epsilon$ in the FitzHugh-Nagumo equation (1) as a small parameter. In [15, we derived a singular bifurcation diagram which represents several important bifurcation curves in $(p, s)$-parameter space in the singular limit $\epsilon=0$. The singular limits of the Hopf and homoclinic curves are shown in Figure 1 as dotted lines 1 In the singular limit, there is no gap between the Hopf and homoclinic curves. We demonstrate below in Proposition 2.1 that a gap must appear for $\epsilon>0$. The main point of this paper is that the termination point of the C-curve at the end of the gap is due to a fast-slow "bifurcation" where the two dimensional stable manifold of an equilibrium is tangent to the two dimensional unstable manifold of a one dimensional slow manifold ${ }^{2}$ Since the analysis of [4] does not explicitly consider slow manifolds of the system, this tangency does not appear in their list of possibilities for the termination of a C-curve. Note that the slow manifolds of the system are unique only up to "exponentially small" quantities of the form $\exp (-c / \epsilon), c>0$, so our analysis only identifies the termination point up to exponentially small values of the parameters.

Fast-slow dynamical systems can be written in the form

$$
\begin{aligned}
\epsilon \dot{x} & =\epsilon \frac{d x}{d \tau}=f(x, y, \epsilon) \\
\dot{y} & =\frac{d y}{d \tau}=g(x, y, \epsilon)
\end{aligned}
$$

where $(x, y) \in \mathbb{R}^{m} \times \mathbb{R}^{n}$ and $\epsilon$ is a small parameter $0<\epsilon \ll 1$. The functions $f: \mathbb{R}^{m} \times \mathbb{R}^{n} \times \mathbb{R} \rightarrow \mathbb{R}^{m}$ and $g: \mathbb{R}^{m} \times \mathbb{R}^{n} \times \mathbb{R} \rightarrow \mathbb{R}^{n}$ are analytic in the systems studied in this paper. The variables $x$ are fast and the

\footnotetext{
${ }^{1}$ In Section 2 we recall the precise meaning of the singular limit bifurcation from [15] and how they these bifurcations arise when $\epsilon=0$.

${ }^{2} \mathrm{An}$ analogous tangency plays an important role in the formation of mixed mode oscillations associated with singular Hopf bifurcations in fast-slow systems with one fast and two slow variables [12].
} 
variables $y$ are slow. We can change (2) from the slow time scale $\tau$ to the fast time scale $t=\tau / \epsilon$, yielding

$$
\begin{aligned}
& x^{\prime}=\frac{d x}{d t}=f(x, y, \epsilon) \\
& y^{\prime}=\frac{d y}{d t}=\epsilon g(x, y, \epsilon)
\end{aligned}
$$

In the singular limit $\epsilon \rightarrow 0$ the system (2) becomes a differential-algebraic equation. The algebraic constraint defines the critical manifold:

$$
C_{0}=\left\{(x, y) \in \mathbb{R}^{m} \times \mathbb{R}^{n}: f(x, y, 0)=0\right\}
$$

For a point $p \in C_{0}$ we say that $C_{0}$ is normally hyperbolic at $p$ if the all the eigenvalues of the $m \times m$ matrix $D_{x} f(p)$ have non-zero real parts. A normally hyperbolic subset of $C_{0}$ is an actual manifold and we can locally parametrize it by a function $h(y)=x$. This yields the slow subsystem (or reduced flow) $\dot{y}=g(h(y), y)$ defined on $C_{0}$. Taking the singular limit $\epsilon \rightarrow 0$ in (3) gives the fast subsystem (or layer equations) $x^{\prime}=f(x, y)$ with the slow variables $y$ acting as parameters. Fenichel's Theorem 9 states that normally hyperbolic critical manifolds perturb to invariant slow manifolds $C_{\epsilon}$. A slow manifold $C_{\epsilon}$ is $O(\epsilon)$ distance away from $C_{0}$. The flow on the (locally) invariant manifold $C_{\epsilon}$ converges to the slow subsystem on the critical manifold as $\epsilon \rightarrow 0$. Slow manifolds are usually not unique for a fixed value of $\epsilon=\epsilon_{0}$ but lie at a distance $O\left(e^{-K / \epsilon_{0}}\right)$ away from each other for some $K>0$; nevertheless we shall refer to "the slow manifold" for a fast-slow system with the possibility of an exponentially small error being understood.

Section 2 discusses the fast-slow decomposition of the homoclinic orbits of the FitzHugh-Nagumo equation in the region $I$. This decomposition has been used to prove the existence of homoclinic orbits in the system for $\epsilon$ sufficiently small [2, 17, 21, 20, 25, but previous work only applies to a situation where the equilibrium point for the homoclinic orbit is not close to a fold point. At a fold point the critical manifold of a fast-slow system is locally quadratic and not normally hyperbolic. This new aspect of the decomposition is key to understanding the sharp turn of the homoclinic curve. Section 3 presents a numerical study that highlights the geometric mechanism for the turning of the C-curve. We visualize relevant aspects of the phase portraits near the turns of the C-curve. In Section 4 we show that exponential contraction of the Shilnikov return map in the FitzHugh-Nagumo equation explains why n-homoclinic and n-periodic orbits are expected to be found at parameter values very close to a primary 1-homoclinic orbit. Section 5 presents two further observations. We identify where a canard explosion [26] occurs and we note the existence of two different types of mixed-mode oscillations in the system.

\section{Fast-Slow Decomposition of Homoclinic Orbits}

We introduce notation used in our earlier work [15. The critical manifold of (1) is given by:

$$
C_{0}=\left\{\left(x_{1}, x_{2}, y\right) \in \mathbb{R}^{3}: x_{2}=0 \text { and } y=f\left(x_{1}\right)+p\right\}
$$

It is normally hyperbolic away from the two fold points $x_{1, \pm}$ with $x_{1,-}<x_{1,+}$ which are found by solving $f^{\prime}\left(x_{1}\right)=0$ as the local minimum and maximum of the cubic $f$. Hence $C_{0}$ splits into three parts:

$$
C_{l}=\left\{x_{1}<x_{1,-}\right\} \cap C_{0}, \quad C_{m}=\left\{x_{1,-} \leq x_{1} \leq x_{1,+}\right\} \cap C_{0}, \quad C_{r}=\left\{x_{1,+}\right\} \cap C_{0}
$$

We are mostly interested in the two branches $C_{l}$ and $C_{r}$ which are of saddle-type, i.e. points in $C_{l}$ and $C_{r}$ are saddle equilibria of the fast subsystem. The middle branch $C_{m}-\left\{x_{1, \pm}\right\}$ consists of unstable foci for the fast subsystem. The slow manifolds provided by Fenichel's Theorem will be denoted by $C_{l, \epsilon}$ and $C_{r, \epsilon}$. The notation for the two-dimensional stable and unstable manifolds of $C_{l, \epsilon}$ is $W^{s}\left(C_{l, \epsilon}\right)$ and $W^{u}\left(C_{r, \epsilon}\right)$ with similar notation for $C_{r, \epsilon}$; the notation for the associated linear eigenspaces is e.g. $E^{s}\left(C_{l, \epsilon}\right)$. The full system (1) has a unique equilibrium point which we denote by $q$. For $(p, s) \in I$ and $\epsilon=0.01$ the dimensions of the stable and unstable manifolds are $\operatorname{dim}\left(W^{u}(q)\right)=1$ and $\operatorname{dim}\left(W^{s}(q)\right)=2$ with a complex conjugate pair of eigenvalues for the linearization at $q$. The equilibrium $q$ is completely unstable inside the U-curve and the Hopf bifurcations we are interested in near $I$ are all subcritical [15, 4]. 
As $\epsilon \rightarrow 0$ the Hopf bifurcation curve converges to a region in $(p, s)$ parameter space bounded by two vertical lines $p=p_{ \pm}$and the segment $\left\{s=0, p_{-} \leq p \leq p_{+}\right\}$; see Figure 1. The parameter values $p_{ \pm}$are precisely the values when the equilibrium point $q$ coincides with the fold points $x_{1, \pm}$ [15]. This analysis gives one part of the singular limit bifurcation diagram showing what happens to the Hopf bifurcation curves for $\epsilon=0$.

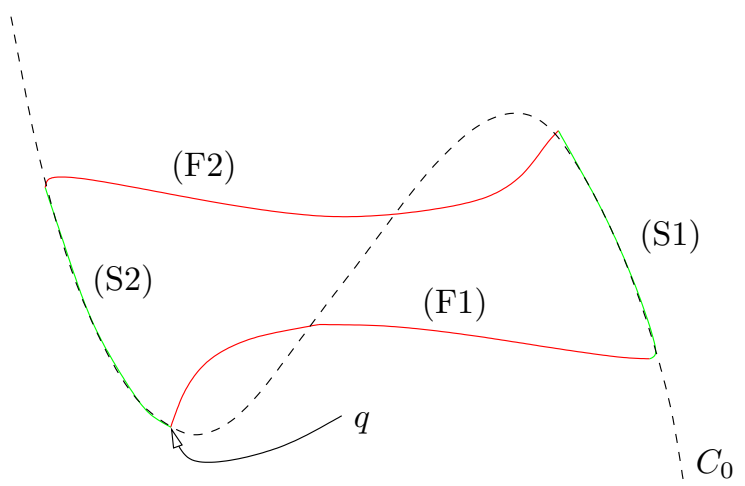

Figure 2: Sketch of a homoclinic orbit to the unique equilibrium q. Fast (red) and slow (green) segments decompose the orbit into segments.

When $\epsilon$ is small, the homoclinic orbit in $W^{u}(q) \cap W^{s}(q)$ can be partitioned into fast and slow segments. The singular limit of this fast-slow decomposition has four segments: a fast subsystem heteroclinic connection from $q$ to $C_{r}$, a slow segment on $C_{r}$, a heteroclinic connection from $C_{r}$ to $C_{l}$ and a slow segment back to $q$ on $C_{l}$; see Figure 2. Existence proofs for the homoclinic orbits [20, 17, 2] are based upon analysis of the transitions between these segments. Trajectories that remain close to a normally hyperbolic slow manifold must be "exponentially close" to the manifold except for short segments where the trajectory approaches the slow manifold along its stable manifold and departs along its unstable manifold. Existence of the homoclinic orbit depends upon how the four segments of its fast-slow decomposition fit together:

(F1) The one dimensional $W^{u}(q)$ approaches $C_{r}$ along its two dimensional stable manifold $W^{s}\left(C_{r, \epsilon}\right)$. Intersection of these manifolds cannot be transverse and occurs only for parameter values that lie along a curve in the $(p, s)$ parameter plane.

(S1) The Exchange Lemma [19] was developed to analyze the flow map for trajectories that approach $C_{r, \epsilon}$ along its stable manifold and depart $C_{r, \epsilon}$ along its unstable manifold.

(F2) The fast jump from a neighborhood of $C_{r, \epsilon}$ to a neighborhood of $C_{l, \epsilon}$ occurs along a transversal intersection of the two dimensional $W^{s}\left(C_{l, \epsilon}\right)$ and two dimensional $W^{u}\left(C_{r, \epsilon}\right)$.

(S2) The connection from $C_{l, \epsilon}$ to $q$ lies close to an intersection of the two dimensional $W^{u}\left(C_{l, \epsilon}\right)$ and the two dimensional $W^{s}(q)$. Previous analysis has dealt with parameter regions where the connection (S2) exists and is transversal, but it cannot persist up to the Hopf curve in the $(p, s)$-plane.

Proposition 2.1. There exists a region in $(p, s)$-parameter space near the Hopf U-curve where no trajectories close to $C_{l, \epsilon}$ lie in $W^{s}(q)$.

Proof. (Sketch) The Lyapunov coefficients of the Hopf bifurcations near $I$ are positive [15], so the periodic orbits emanating from these bifurcations occur in the parameter region to the left of the Hopf curve. The periodic orbits are completely unstable. By calculating the eigenvalues of the linearization at the equilibrium we find that there is no Fold-Hopf bifurcation on the Hopf curve near $I$. Hence center manifold reduction implies that there will be a region of parameters near the Hopf curve where $W^{s}(q)$ is a topological disk whose boundary is the periodic orbit. Close enough to the Hopf curve, $W^{s}(q)$ and the periodic orbit lie at a finite distance from $C_{l, \epsilon}$ and there is no connection from $C_{l, \epsilon}$ to $q$. 
This proposition implies that the parameter region in which there is a connection from $C_{l, \epsilon}$ to $q$ is bounded away from the Hopf curve. The next section shows that the boundary of this parameter region is very close to a curve along which there are tangential intersections of $W^{u}\left(C_{l, \epsilon}\right)$ and $W^{s}(q)$.

Remark: As $\epsilon \rightarrow 0$, the C-curve converges to two lines (dashed red in Figure1) defined by homoclinic and heteroclinic orbits of the fast subsystem [15]. The horizontal segment of the C-curve to homoclinic orbits of the equilibrium point, and the sloped segment to heteroclinic orbits from the equilibrium point to the right branch of the critical manifold. Note that the C-curve terminates on the Hopf curve in the singular limit. The singular limit analysis does not explain the sharp turning of the C-curve for $\epsilon>0$ which is the focus of the next section.

\section{Interaction of Invariant Manifolds}

The slow manifold $C_{l, \epsilon}$ is normally hyperbolic away from the fold point $x_{1,-}$, with one attracting direction and one repelling direction. We recently introduced a method [14] for computing slow manifolds of saddle type. This algorithm is used here to help determine whether there are connecting orbits from a neighborhood of $C_{l, \epsilon}$ to the equilibrium point $q$. Our numerical strategy for finding connecting orbits has three steps:

1. Choose the cross section

$$
\Sigma_{0.09}=\left\{\left(x_{1}, x_{2}, y\right) \in \mathbb{R}^{3}: y=0.09\right\}
$$

transverse to $C_{l, \epsilon}$,

2. Compute intersections of trajectories in $W^{s}(q)$ with $\Sigma_{0.09}$. These points are found either by backward integration from initial conditions that lie in a small disk $D$ containing $q$ in $W^{s}(q)$ or by solving a boundary value problem for trajectories that have one end in $\Sigma_{0.09}$ and one end on the boundary of $D$.

3. Compute the intersection $p_{l} \in C_{l, \epsilon} \cap \Sigma_{0.09}$ with the algorithm described in Guckenheimer and Kuehn [14] and determine the directions of the positive and negative eigenvectors of the Jacobian of the fast subsystem at $p_{l}$.

Figure 3 shows the result of these computations for $\epsilon=0.01, s=1.37$ and three values of $p 3^{3}$ The intersections of $W^{s}(q)$ with $\Sigma_{0.09}$ lies close to $W^{s}\left(C_{l, \epsilon}\right)$.

\begin{tabular}{|c|c|}
\hline$\epsilon$ & $\mathrm{D}=\mathrm{d}($ tangency,Hopf $)$ \\
\hline $10^{-2}$ & $\approx 1.07 \epsilon$ \\
$10^{-3}$ & $\approx 1.00 \epsilon$ \\
$10^{-4}$ & $\approx 0.98 \epsilon$ \\
\hline
\end{tabular}

Table 1: Euclidean distance in $(\mathrm{p}, \mathrm{s})$-parameter space between the Hopf curve and the location of the tangency point between $W^{s}(q)$ and $W^{u}\left(C_{l, \epsilon}\right)$.

Backward trajectories flowing along $C_{l, \epsilon}$ converge to its stable manifold at a fast exponential rate. This fact also explains the observation that $W^{s}(q) \cap \Sigma_{0.09}$ makes a sharp turn. In Figure 3(a), it is apparent that the turn lies to the left of $W^{u}\left(C_{l, \epsilon}\right) \cap \Sigma_{0.09}$ and that $W^{s}(q) \cap W^{u}\left(C_{l, \epsilon}\right)$ is non-empty. In Figure 3(c), the turn lies to the right of $W^{u}\left(C_{l, \epsilon}\right) \cap \Sigma_{0.09}$. We have also computed the distance from the Hopf curve of the parameters at which $W^{s}(q)$ and $W^{u}\left(C_{l, \epsilon}\right)$ appear to have a tangential intersection for several different values of $\epsilon$; see Table 1 from which we observe that the distance is $O(\epsilon)$.

\footnotetext{
${ }^{3}$ The second step above was carried out with two different initial value solvers, ode15s in Matlab [31] and dop853 [16], and with AUTO 7] used as a boundary value solver. All three methods produced similar results.
} 


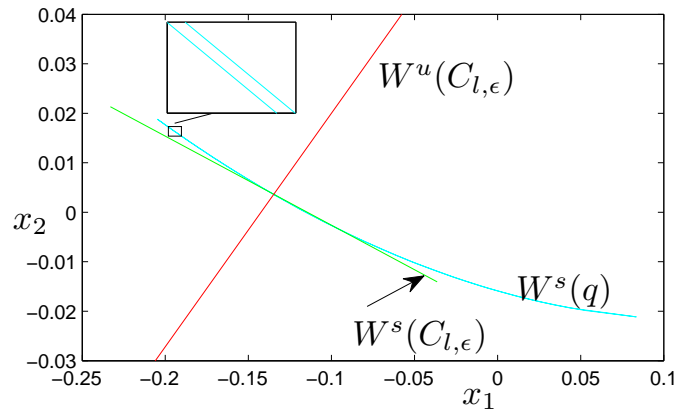

(a) $p=0.059, s=1.37$

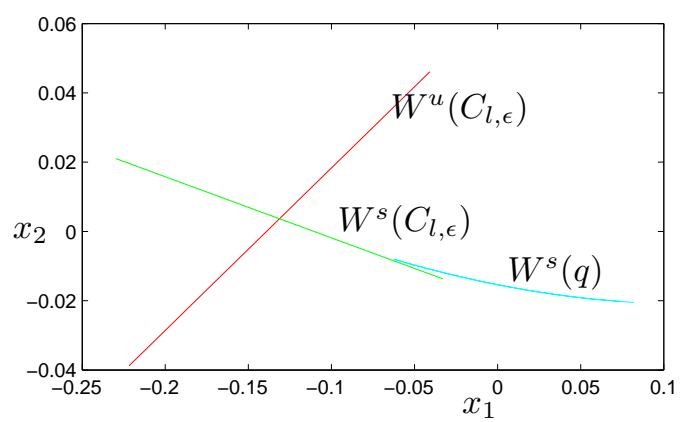

(c) $p=0.06, s=1.37$

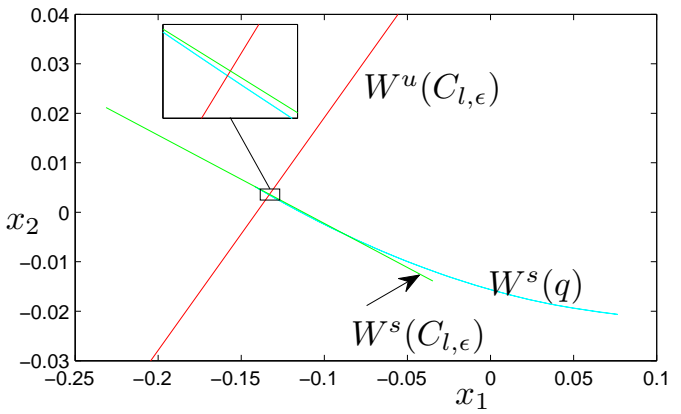

(b) $p=0.0595, s=1.37$

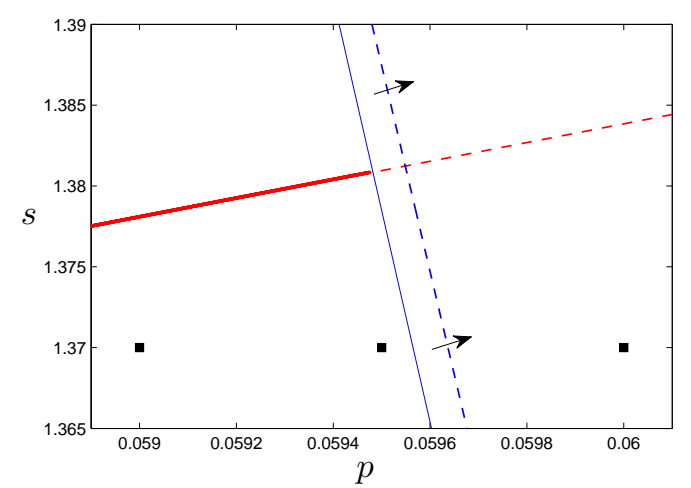

(d) Parameter space, region $I$.

Figure 3: Figures (a)-(c) show the movement of the stable manifold $W^{s}(q)$ (cyan) with respect to $E^{u}\left(C_{l, \epsilon}\right)$ (red) and $E^{s}\left(C_{l, \epsilon}\right)$ (green) in phase space on the section $y=0.09$ for $\epsilon=0.01$. The parameter space diagram (d) shows the homoclinic C-curve (solid red), an extension of the C-curve of parameters where $W^{u}(q) \cap W^{s}\left(C_{r, \epsilon}\right)$ is nonempty, a curve that marks the tangency of $W^{s}(q)$ to $E^{u}\left(C_{l, \epsilon}\right)$ (blue) and a curve that marks a distance between $C_{l, \epsilon}$ and $W^{s}(q)$ (dashed blue) of 0.01 where the arrows indicate the direction in which the distance is bigger than 0.01. The solid black squares in (d) show the parameter values for (a)-(c). 
In Figure 3(d) the C-curve of homoclinic bifurcations (solid red) has been computed using continuation in AUTO [7] as carried out by Champneys et al. [4. Despite the fact that no homoclinic orbit exists in part of the region $I$ it is possible to check whether the unstable manifold $W^{u}(q)$ reaches a small neighborhood of $W^{s}\left(C_{r, \epsilon}\right)$. This idea has been used in a splitting algorithm [15 to calculate where homoclinic orbits would occur if $W^{s}(q)$ would not move away from $C_{l, \epsilon}$ as shown in Figures $3(\mathrm{a})-3$ (d). This yields the dashed red curve in Figure 3(d). On this curve we verified that $W^{s}\left(C_{l, \epsilon}\right)$ and $W^{u}\left(C_{r, \epsilon}\right)$ still intersect transversally by computing those manifolds; see [15, 14, for details.

The blue curves in Figure 3(d) have been obtained by measuring the Euclidean distances between $W^{s}(q)$ and $C_{l, \epsilon}$ in the section $\Sigma_{0.09}$. Along the dashed blue curve the distance between $C_{l, \epsilon}$ and $W^{s}(q)$ is 0.01 . The arrows indicate the direction in which this distance increases. The solid blue curve marks a tangency of $W^{s}(q)$ with $E^{u}\left(C_{l, \epsilon}\right)$. These calculations demonstrate that the sharp turn in the C-curve of homoclinic bifurcations occurs very close to the curve where there is a tangential intersection of $W^{s}(q)$ and $W^{u}\left(C_{l, \epsilon}\right)$. Therefore, we state the following conjecture.

Conjecture 3.1. The C-curve of homoclinic bifurcations of the FitzHugh-Nagumo system turns exponentially close to the boundary of the region where $W^{u}\left(C_{l, \epsilon}\right) \cap W^{s}(q)$ is nonempty.

Note that trajectory segments of types (F1), (S1) and (F2) are still present along the dashed red curve in Figure 3(d). Only the last slow connection (S2) no longer exists. Existence proofs for homoclinic orbits that use Fenichel's Theorem for $C_{l}$ to conclude that trajectories entering a small neighborhood of $C_{l, \epsilon}$ must intersect $W^{s}(q)$ break down in this region. The equilibrium $q$ has already moved past the fold point $x_{1,-}$ in $I$ as seen from the singular bifurcation diagram in Figure 1 where the blue dashed vertical lines mark the parameter values where $q$ passes through $x_{1, \pm}$. Therefore Fenichel's Theorem does not provide the required perturbation of $C_{l, \epsilon}$. Previous proofs [20, 17, 2] assumed $p=0$ and the connecting orbits of type (S2) do exist in this case.

Shilnikov proved that there are chaotic invariant sets in the neighborhood of homoclinic orbits to a saddle-focus in three dimensional vector fields when the magnitude of the real eigenvalue is larger than the magnitude of the real part of the complex pair of eigenvalues [32. The homoclinic orbits of the FitzHughNagumo vector field satisfy this condition in the parameter region $I$. Therefore, we expect to find many periodic orbits close to the homoclinic orbits and parameters in $I$ with "multi-pulse" homoclinic orbits that have several jumps connecting the left and right branches of the slow manifold $[8$. Without making use of concepts from fast-slow systems, Champneys et al. 4 described interactions of homoclinic and periodic orbits that can serve to terminate curves of homoclinic bifurcations. This provides an alternate perspective on identifying phenomena that occur near the sharp turn of the C-curve in $I$. AUTO can be used to locate families of periodic orbits that come close to a homoclinic orbit as their periods grow.

Figure 4 shows several significant objects in phase space for parameters lying on the C-curve. The homoclinic orbit and the two periodic orbits were calculated using AUTO. The periodic orbits were continued in $p$ starting from a Hopf bifurcation for fixed $s \approx 1.3254$. Note that the periodic orbit undergoes several fold bifurcations [4. We show two of the periodic orbits arising at $p=0.05$; see [4. The trajectories in $W^{s}\left(C_{l, \epsilon}\right)$ have been calculated using a mesh on $C_{l, \epsilon}$ and using backward integration at each mesh point and initial conditions in the linear approximation $E^{s}\left(C_{l, \epsilon}\right)$.

We observe from Figure 4 that part of $W^{s}(q)$ lies near $C_{l, \epsilon}$ as expected for (S2) to be satisfied. This is in contrast to the situation beyond the turning of the C-curve shown in Figure 5 for $p=0.06$ and $s=1.38$. We observe that $W^{s}(q)$ is bounded. Figure 5 (a) shows two periodic orbits $P_{1}$ and $P_{2}$ obtained from a Hopf bifurcation continuation starting for $s=1.38$ fixed. $P_{2}$ is of large amplitude and is obtained after the first fold bifurcation occurred. $P_{1}$ is of small amplitude and is completely unstable. A zoom near $P_{1}$ in Figure 5 (b) and a time series comparison of a trajectory in $W^{s}(q)$ and $P_{1}$ in Figure 5(c) show that

$$
\lim _{\alpha}\left\{p: p \in W^{s}(q) \text { and } p \neq q\right\}=P_{1}
$$




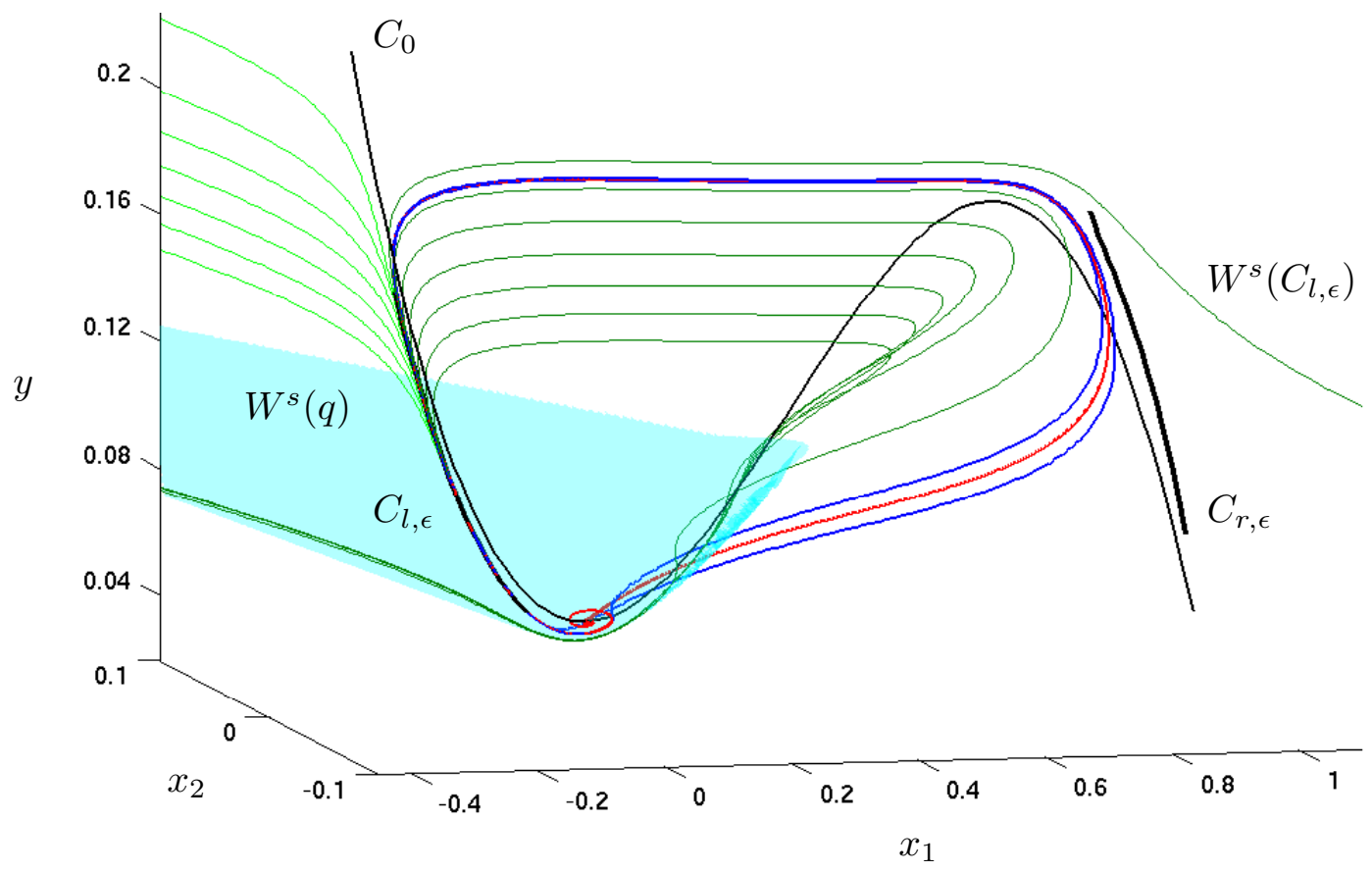

Figure 4: Phase space along the C-curve near its sharp turn: the parameter values $\epsilon=0.01, p=0.05$ and $s \approx 1.3254$ lie on the C-curve. The homoclinic orbit (red), two periodic orbits born in the subcritical Hopf (blue), $C_{0}$ (thin black), $C_{l, \epsilon}$ and $C_{r, \epsilon}$ (thick black) are shown. The manifold $W^{s}(q)$ (cyan) has been truncated at a fixed coordinate of $y$. Furthermore $W^{s}\left(C_{l, \epsilon}\right)$ (green) is separated by $C_{l, \epsilon}$ into two components shown here by dark green trajectories interacting with $C_{m, \epsilon}$ and by light green trajectories that flow left from $C_{l, \epsilon}$.

where $\lim _{\alpha} U$ denotes the $\alpha$-limit set of some set $U \subset \mathbb{R}^{m} \times \mathbb{R}^{n}$. From (4) we can also conclude that there is no heteroclinic connection from $q$ to $P_{1}$ and only a connection from $P_{1}$ to $q$ in a large part of the region $I$ beyond the turning of the C-curve. Since $P_{1}$ is completely unstable, there can be no heteroclinic connections from $q$ to $P_{1}$. Therefore, double heteroclinic connections between a periodic orbit and $q$ are restricted to periodic orbits that lie closer to the homoclinic orbit than $P_{1}$. These can be expected to exist for parameter values near the end of the C-curve in accord with the conjecture of Champneys et al. [4 and the "Shilnikov"model presented in the next section.

Remark: The recent manuscript [3] extends the results of [4 that motivated this paper. A partial unfolding of a heteroclinic cycle between a hyperbolic equilibrium point and a hyperbolic periodic orbit is developed in [3]. Champneys et al. call this codimension two bifurcation an EP1t-cycle and the point where it occurs in a two dimensional parameter space an EP1t-point. The manuscript [3] does not conclude whether the EP1t-scenario occurs in the FitzHugh-Nagumo equation. The relationship between the results of this paper and those of [3] have not yet been clarified.

\section{Homoclinic Bifurcations in Fast-Slow Systems}

It is evident from Figure 3 that the homoclinic orbits in the FitzHugh-Nagumo equation exist in a very thin region in $(p, s)$-parameter space along the $\mathrm{C}$-curve. We develop a geometric model for homoclinic orbits that resemble those in the FitzHugh-Nagumo equation containing segments of types (S1), (F1), (S2) and (F2). The model will be seen to be an exponentially distorted version of the Shilnikov model for a homoclinic orbit to a saddle-focus [13. Throughout this section we assume that the parameters lie in a region $I$ the region of the $(p, s)$-plane close to the upper turn of the C-curve.

The return map of the Shilnikov model is constructed from two components: the flow map past an 


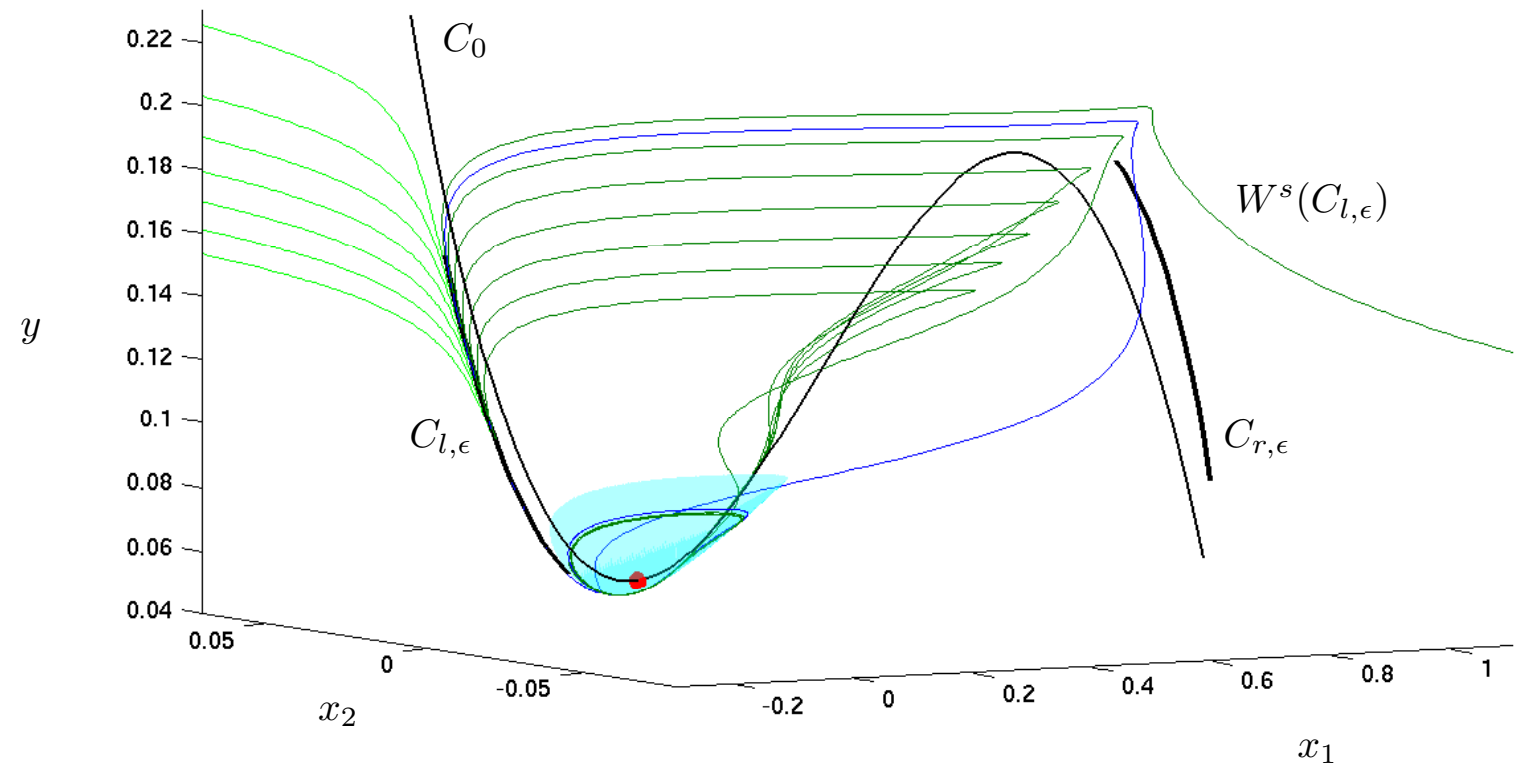

(a) Sample phase space plot between the end of the C-curve and the U-curve.

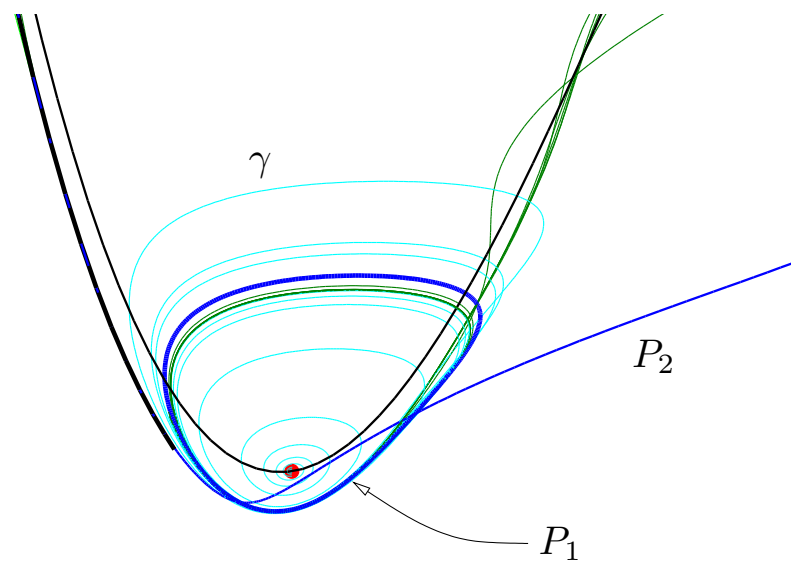

(b) Zoom for (a) near $q$.

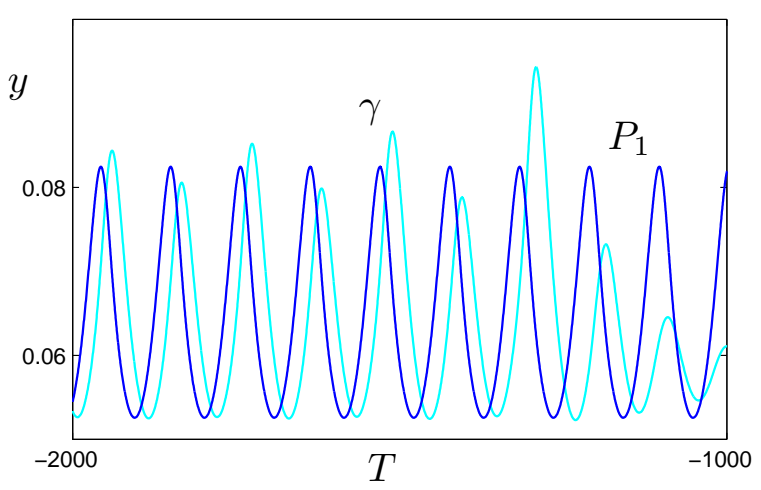

(c) Time series.

Figure 5: The parameter values are $\epsilon=0.01, p=0.06$ and $s=1.38$. For (a) we display two periodic orbits (blue), one with a single large excursion $P_{2}$ and one consisting of a small loop $P_{1}$. We also show $q$ (red dot), trajectories in $W^{s}\left(C_{l, \epsilon}\right)$ (green) and $W^{s}(q)$ (cyan). In (b) a zoom near $q$ is shown and we made plotted a single trajectory $\gamma \in W^{s}(q)$ (cyan). The plot (c) gives a time series of this trajectory $\gamma$ in comparison to the periodic orbit $P_{1}$. Note that the trajectories are computed backward in time, so the final points of the trajectories are on the left of the figure. A phase shift of time along the periodic orbit would bring the two time series closer. 
equilibrium point, approximated by the flow map of a linear vector field, composed with a regular map that gives a "global return" of the unstable manifold of the equilibrium to its stable manifold [13]. Place two cross-sections $\Sigma_{1}$ and $\Sigma_{2}$ moderately close to the equilibrium point and model the flow map from $\Sigma_{1}$ to $\Sigma_{2}$ via the linearization of the vector field at the equilibrium.

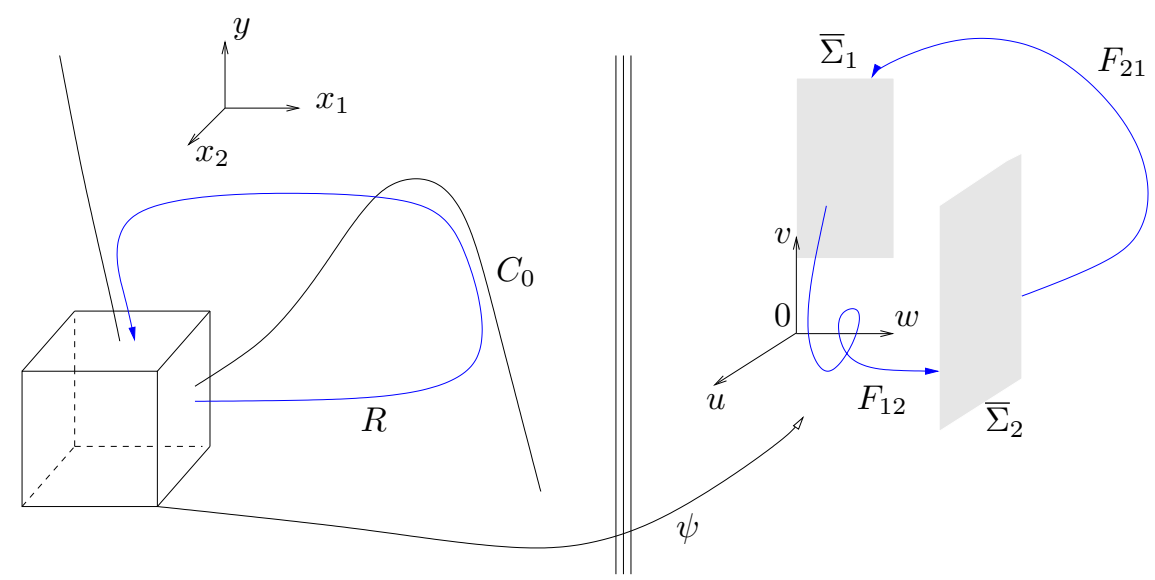

Figure 6: Sketch of the geometric model for the homoclinic bifurcations. Only parts of the sections $\bar{\Sigma}_{i}$ for $i=1,2$ are shown.

The degree one coefficient of the characteristic polynomial at the equilibrium has order $O(\epsilon)$, so the imaginary eigenvalues at the Hopf bifurcation point have magnitude $O\left(\epsilon^{1 / 2}\right)$. The real part of these eigenvalues scales linearly with the distance from the Hopf curve. Furthermore we note that the real eigenvalue of the equilibrium point remains bounded away from 0 as $\epsilon \rightarrow 0$.

Let $\psi\left(x_{1}, x_{2}, y\right)=(u, v, w)$ be a coordinate change near $q$ so that $\psi(q)=0$ and the vector field is in Jordan normal form up to higher order terms. We denote the sections obtained from the coordinate change into Jordan form coordinates by $\bar{\Sigma}_{1}=\psi\left(\Sigma_{1}\right)$ and $\bar{\Sigma}_{2}=\psi\left(\Sigma_{2}\right)$; see Figure 6 . Then the vector field is

$$
\begin{aligned}
u^{\prime} & =-\beta u-\alpha v \\
v^{\prime} & =\alpha u-\beta v \quad \text { +h.o.t. } \\
w^{\prime} & =\gamma
\end{aligned}
$$

with $\alpha, \beta, \gamma$ positive. We can choose $\psi$ so that the cross-sections are $\bar{\Sigma}_{1}=\{u=0, w>0\}$ and $\bar{\Sigma}_{2}=\{w=1\}$. The flow map $F_{12}: \bar{\Sigma}_{1} \rightarrow \bar{\Sigma}_{2}$ of the (linear) vector field (5) without higher-order terms is given by

$$
F_{12}(v, w)=v w^{\beta / \gamma}\left(\cos \left(-\frac{\alpha}{\gamma} \ln (w)\right), \sin \left(-\frac{\alpha}{\gamma} \ln (w)\right)\right)
$$

Here $\beta$ and $\alpha$ tend to 0 as $\epsilon \rightarrow 0$. The domain for $F_{12}$ is restricted to the interval $v \in[\exp (-2 \pi \beta / \alpha), 1]$ bounded by two successive intersections of a trajectory in $W^{s}(0)$ with the cross-section $u=0$.

The global return map $R: \Sigma_{2} \rightarrow \Sigma_{1}$ of the FitzHugh-Nagumo system is obtained by following trajectories that have successive segments that are near $W^{s}\left(C_{r, \epsilon}\right)$ (fast), $C_{r, \epsilon}$ (slow), $W^{u}\left(C_{r, \epsilon}\right) \cap W^{s}\left(C_{l, \epsilon}\right)$ (fast), $C_{l, \epsilon}$ (slow) and $W^{u}\left(C_{l, \epsilon}\right)$ (fast). The Exchange Lemma [19] implies that the size of the domain of $R$ in $\Sigma_{2}$ is a strip whose width is exponentially small. As the parameter $p$ is varied, we found numerically that the image of $R$ has a point of quadratic tangency with $W^{s}(q)$ at a particular value of $p$. We also noted that $W^{u}(q)$ crosses $W^{s}\left(C_{r, \epsilon}\right)$ as the parameter $s$ varies [15]. Thus, we choose to model $R$ by the map

$$
(w, v)=F_{21}(u, v)=\left(\sigma v+\lambda_{2}-\rho^{2}\left(u-\lambda_{1}\right)^{2}, \rho\left(u-\lambda_{1}\right)+\lambda_{3}\right)
$$

for $F_{21}$ where $\lambda_{1}$ represents the distance of $W^{u}(q) \cap \Sigma_{2}$ from the domain of $F_{21}, \lambda_{2}$ represents how far the image of $F_{21}$ extends in the direction normal to $W^{s}(q), \lambda_{3}$ is the $v$ coordinate of $F_{21}\left(\lambda_{1}, 0\right)$ and $\rho^{-1}, \sigma$ are 
$O\left(e^{-K / \epsilon}\right)$ for suitable $K>0$. We assume further that the domain of $F_{21}$ is $\left[\lambda_{1}, \lambda_{1}+\rho^{-1}\right] \times[-1,1]$. Figure 7 depicts $F_{21}$. With these choices, we observe two properties of the C-curve of homoclinic orbits in the geometric model:

1. If $\sigma v+\lambda_{2}-\rho^{2}\left(u-\lambda_{1}\right)^{2}$ is negative on the domain of $F_{21}$, then the image of $F_{21}$ is disjoint from the domain of $F_{12}$ and there are no recurrent orbits passing near the saddle point. Thus, recurrence implies that $\lambda_{2}>-\sigma$.

2. If $\lambda_{2}>0$, then there are two values of $\lambda_{1}$ for which the saddle-point has a single pulse homoclinic orbit. These points occur for values of $\lambda_{1}$ for which the $w$-component of $F_{21}(0,0)$ vanishes: $\lambda_{1}= \pm \rho^{-1}\left|\lambda_{2}\right|^{1 / 2}$. The magnitude of these values of $\lambda_{2}$ is exponentially small.
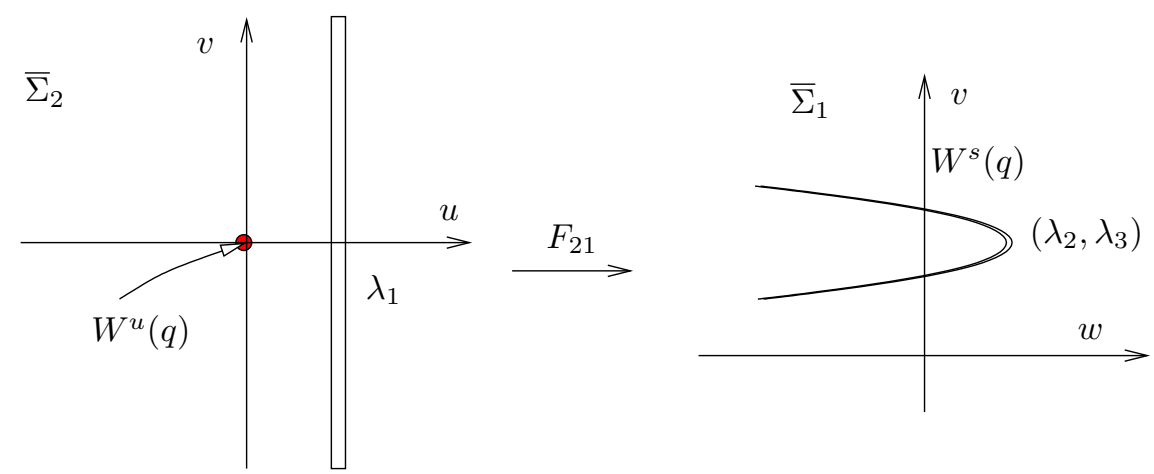

Figure 7: Sketch of the map $F_{21}: \bar{\Sigma}_{2} \rightarrow \bar{\Sigma}_{1}$. The $(u, v)$ coordinates are centered at $W^{u}(q)$ and the domain of $F_{21}$ is in the thin rectangle at distance $\lambda_{1}$ from the origin. The image of this rectangle is the parabolic strip in $\bar{\Sigma}_{1}$.

When a vector field has a single pulse homoclinic orbit to a saddle-focus whose real eigenvalue has larger magnitude than the real part of the complex eigenvalues, Shilnikov 32 proved that a neighborhood of this homoclinic orbit contains chaotic invariant sets. This conclusion applies to our geometric model when it has a single pulse homoclinic orbit. Consequently, there will be a plethora of bifurcations that occur in the parameter interval $\lambda_{2} \in[0, \sigma]$, creating the invariant sets as $\lambda_{2}$ decreases from $\sigma$ to 0 .

The numerical results in the previous section suggest that in the FitzHugh-Nagumo system, some of the periodic orbits in the invariant sets near the homoclinic orbit can be continued to the Hopf bifurcation of the equilibrium point. Note that saddle-node bifurcations that create periodic orbits in the invariant sets of the geometric model lie exponentially close to the curve $\lambda_{2}=0$ that models tangency of $W^{s}(q)$ and $W^{u}\left(C_{l, \epsilon}\right)$ in the FitzHugh-Nagumo model. This observation explains why the right most curve of saddle-node bifurcations in Figure 7 of Champneys et al. 4 lies close to the sharp turn of the C-curve.

There will also be curves of heteroclinic orbits between the equilibrium point and periodic orbits close to the C-curve. At least some of these form codimension two EP1t bifurcations near the turn of the C-curve as discussed by Champneys et al. [4. Thus, the tangency between $W^{s}(q)$ and $W^{u}\left(C_{l, \epsilon}\right)$ implies that there are several types of bifurcation curves that pass exponentially close to the sharp turn of the C-curve in the FitzHugh-Nagumo model. Numerically, any of these can be used to approximately locate the sharp turn of the C-curve.

\section{Canards and Mixed Mode Oscillations}

This section reports two additional observations about the FitzHugh-Nagumo model resulting from our numerical investigations and analysis of the turning of the C-curve. 


\subsection{Canard Explosion}

The previous sections draw attention to the intersections of $W^{s}(q)$ and $W^{u}\left(C_{l, \epsilon}\right)$ as a necessary component for the existence of homoclinic orbits in the FitzHugh-Nagumo system. Canards for the backward flow of this system occur along intersections of $W^{u}\left(C_{l, \epsilon}\right)$ and $C_{m, \epsilon}$. These intersections form where trajectories that track $C_{l, \epsilon}$ have continuations that lie along $C_{m, \epsilon}$ which has two unstable fast directions. We observed from Figures 4 and 5 that a completely unstable periodic orbit born in the Hopf bifurcation on the U-curve undergoes a canard explosion, increasing its amplitude to the size of a relaxation oscillation orbit upon decreasing $p$. This canard explosion happens very close to the intersections of $W^{u}\left(C_{l, \epsilon}\right)$ and $C_{m, \epsilon}$.

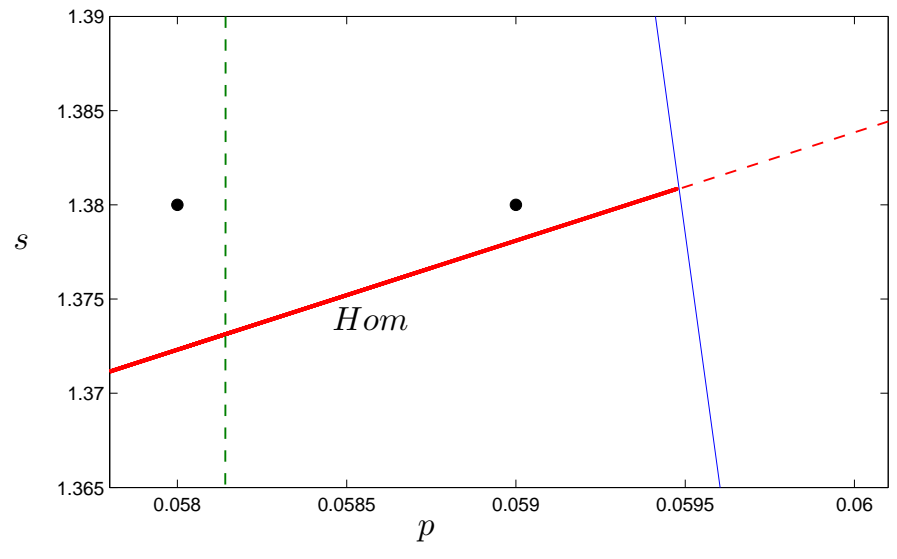

(a) $(p, s)$-space: Black circles correspond to two portraits in (b).
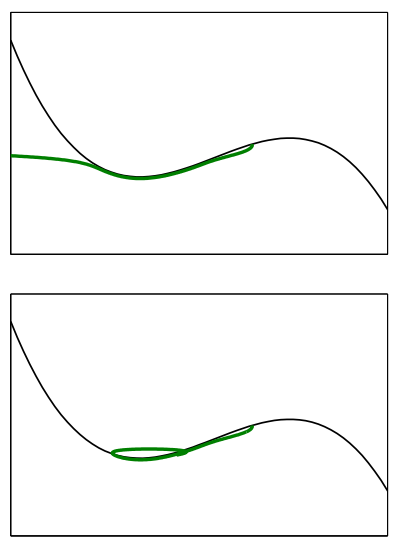

(b) $\left(x_{1}, y\right)$-projection.

Figure 8: The dashed green curve indicates where canard orbits start to occur along $C_{m, \epsilon}$. For values of $p$ to the left of the dashed green curve we observe that orbits near the middle branch escape in backward time (upper panel in (b)). For values of $p$ to the right of the dotted green curve trajectories near $C_{m, \epsilon}$ stay bounded in backward time.

To understand where this transition starts and ends we computed the middle branch $C_{m, \epsilon}$ of the slow manifold by integrating backwards from points between the fold points $x_{1,-}$ and $x_{1,+}$ starting close to $C_{m, 0}$ and determined which side of $W^{u}\left(C_{l, \epsilon}\right)$ these trajectories came from. The results are shown in Figure 8 The dashed green curve divides the $(p, s)$ plane into regions where the trajectory that flows into $C_{m, \epsilon}$ lies to the left of $W^{u}\left(C_{l, \epsilon}\right)$ and is unbounded from the region where the trajectory that flows into $C_{m, \epsilon}$ lies to the right of $W^{u}\left(C_{l, \epsilon}\right)$ and comes from the periodic orbit or another bounded invariant set. This boundary was found by computing trajectories starting on $C_{m, 0}$ backward in time. In backward time the middle branch of the slow manifold is attracting, so the trajectory first approaches $C_{m, \epsilon}$ and then continues beyond its end when $x_{1}$ decreases below $x_{1,-}$. Figure 8 illustrates the difference in the behavior of these trajectories on the two sides of the dashed green curve. It shows that the parameters with canard orbits for the backward flow have smaller values of $p$ than those for which $W^{s}(q)$ and $W^{u}\left(C_{l, \epsilon}\right)$ have a tangential intersection. The turns of the $\mathrm{C}$-curve do not occur at parameters where the backward flow has canards.

\subsection{Mixed-Mode Oscillations}

Mixed-mode oscillations (MMOs) have been observed in many fast-slow systems; see e.g. [28, 29, 30, 12. MMOs are periodic orbits which consist of sequences of small and large amplitude oscillations. The notation $L^{s}$ is used to indicate an MMO with $L$ large and $s$ small oscillations.

The FitzHugh-Nagumo equation (11) exhibits MMOs: the periodic orbits close to the homoclinic orbit make small oscillations near the equilibrium point in addition to large amplitude relaxation oscillations. A $1^{1}$ MMO is shown in Figure 9(a)-(b). It was obtained by switching from the homoclinic C-curve to a nearby 


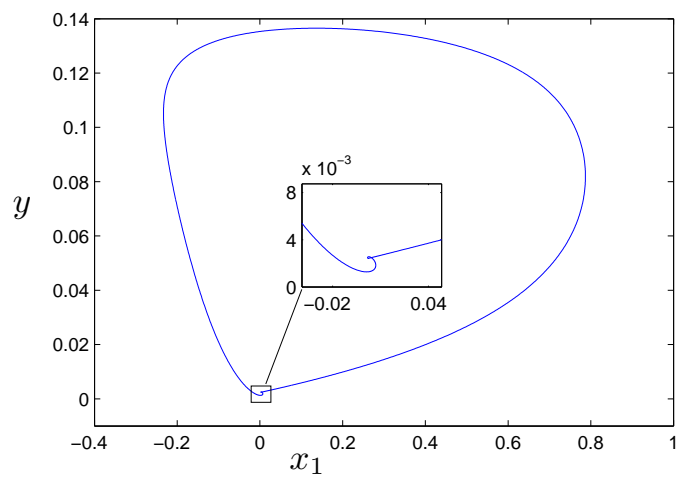

(a) $\left(x_{1}, y\right)$-space, $p=0.00266172$.

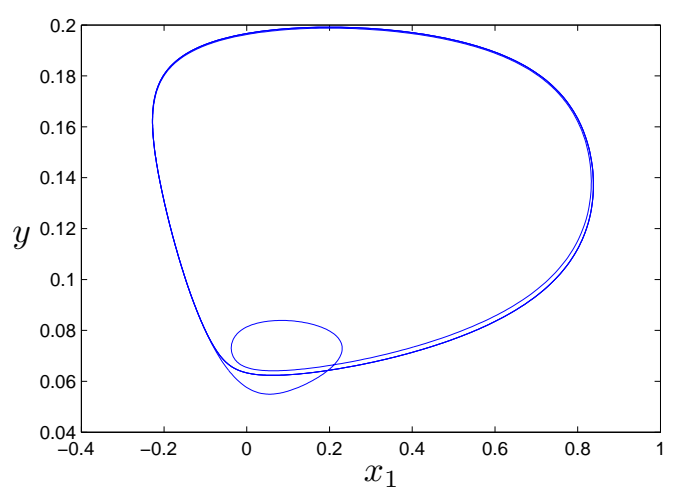

(c) $\left(x_{1}, y\right)$-space, $p=0.0628718$.

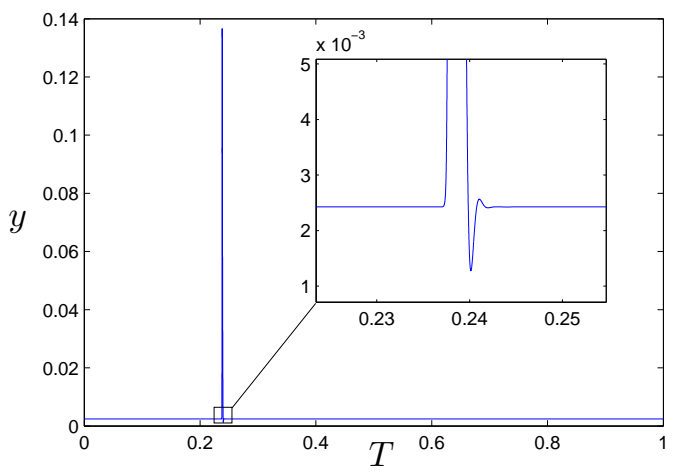

(b) Time series for (a).

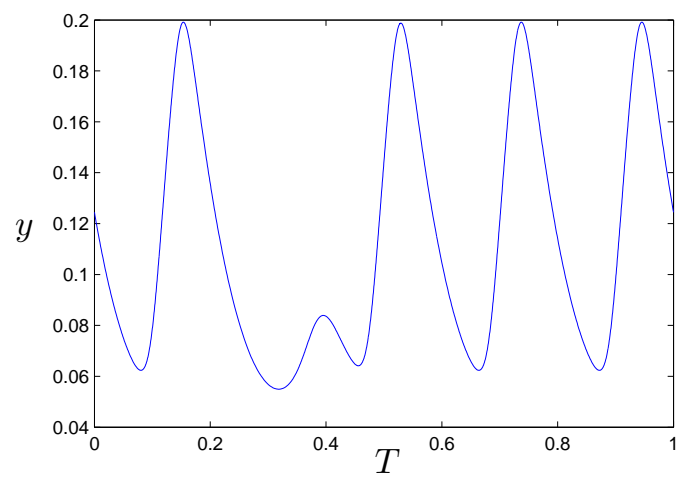

(d) Time Series for (c).

Figure 9: Some examples of mixed-mode oscillations in the FitzHugh-Nagumo equation. Fixed parameter values are $\epsilon=0.01$ and $s=1$. Note that the period of the orbits has been rescaled to 1 in $(b)$ and $(d)$. 
curve of periodic orbits in a continuation framework. Note that the existence of multi-pulse homoclinic orbits near a Shilnikov homoclinic orbit [10, 8] implies that much more complicated patterns of MMOs also exist near the homoclinic C-curve. $L^{s}$ MMOs with very large $L$ and $s$ near the homoclinic C-curve are theoretically possible although observing them will be very difficult due to the exponential contraction described in Section 4

In addition to the MMOs induced by the Shilnikov bifurcation we also find MMOs which exist due to orbits containing canard segments near the completely unstable slow manifold $C_{m, \epsilon}$. An example of a $4^{1}$ MMO is shown in Figure 9(c)-(d) obtained by continuation. In this case the small oscillations arise due to small excursions reminiscent to MMOs in three-time scale systems [18, 24. MMOs of type $L^{1}$ with $L=1,2,3, \ldots, O\left(10^{2}\right)$ can easily be observed from continuation and we expect that $L^{1}$ MMOs exist for any $L \in \mathbb{N}$. It is likely that these MMOs can be analyzed using a version of the FitzHugh-Nagumo equation containing $O(1), O(\epsilon)$ and $O\left(\epsilon^{2}\right)$ terms similar to the one introduced in [15] but we leave this analysis for future work.

Figure 9 was obtained by varying $p$ for fixed values of $\epsilon=0.01$ and $s=1$. Thus, varying a single parameter suffices to switch between MMOs whose small amplitude oscillations have a different character. In the first case, the small amplitude oscillations occur when the orbit comes close to a saddle focus rotating around its stable manifold, while in the second case, the trajectory never approaches the equilibrium and its small amplitude oscillations occur when the trajectory flows along the completely unstable slow manifold $C_{m, \epsilon}$. Different types of MMOs seem to occur very frequently in single- and multi-parameter bifurcation problems; see [6] for a recent example. This contrasts with most work on the analysis of MMOs [22, 29] that focuses on identifying the mechanism for generating MMOs in an example. The MMOs in the FitzHugh-Nagumo equation show that a fast-slow system with three or more variables can exhibit MMOs of different types and that one should not expect a priori that a single mechanism suffices to explain all the MMO dynamics.

\section{References}

[1] D.G. Aronson and H.F. Weinberger. Nonlinear diffusion in population genetics, combustion, and nerve pulse propagation. in: Partial Differential Equations and Related Topics (Lecture Notes in Mathematics), 446:5-49, 1974.

[2] G.A. Carpenter. A geometric approach to singular perturbation problems with applications to nerve impulse equations. Journal of Differential Equations, 23:335-367, 1977.

[3] A.R. Champneys, V. Kirk, E. Knobloch, B.E. Oldeman, and J.D.M. Rademacher. Unfolding a tangent equilibrium-to-periodic heteroclinic cycle. SIAM J. Appl. Dyn. Syst., 8(3):1261-1304, 2009.

[4] A.R. Champneys, V. Kirk, E. Knobloch, B.E. Oldeman, and J. Sneyd. When Shilnikov meets Hopf in excitable systems. SIAM J. Appl. Dyn. Syst., 6(4):663-693, 2007.

[5] M. Desroches, B. Krauskopf, and H.M. Osinga. Mixed-mode oscillations and slow manifolds in the self-coupled FitzHugh-Nagumo system. Chaos, 18, 2008.

[6] M. Desroches, B. Krauskopf, and H.M. Osinga. Numerical continuation of canard orbits in slow-fast dynamical systems. preprint, 2009.

[7] E.J. Doedel et al. Auto 2007p: Continuation and bifurcation software for ordinary differential equations (with homcont). http://cmvl.cs.concordia.ca/auto, 2007.

[8] J.W. Evans, N. Fenichel, and J.A. Feroe. Double impulse solutions in nerve axon equations. SIAM J. Appl. Math., 42(2):219-234, 1982.

[9] N. Fenichel. Geometric singular perturbation theory for ordinary differential equations. Journal of Differential Equations, 31:53-98, 1979. 
[10] S.V. Gonchenko, D.V. Turaev, P. Gaspard, and G. Nicolis. Complexity in the bifurcation structure of homoclinic loops to a saddle-focus. Nonlinearity, 10:409-423, 1997.

[11] W. Govaerts and Yu.A. Kuznetsov. Matcont. http://www.matcont.ugent.be/, 2008.

[12] J. Guckenheimer. Singular Hopf bifurcation in systems with two slow variables. SIAM J. Appl. Dyn. Syst., 7(4):1355-1377, 2008.

[13] J. Guckenheimer and P. Holmes. Nonlinear Oscillations, Dynamical Systems, and Bifurcations of Vector Fields. Springer, 1983.

[14] J. Guckenheimer and C. Kuehn. Computing slow manifolds of saddle-type. SIAM J. Appl. Dyn. Syst., $4(3): 854-879,2009$.

[15] J. Guckenheimer. and C. Kuehn Homoclinic orbits of the FitzHugh-Nagumo equation: The singular limit. Discrete and Continuous Dynamical Systems: Series S, 2(4):851-872, 2009.

[16] E. Hairer and G. Wanner. Solving Ordinary Differential Equations II. Springer, 1991.

[17] S.P. Hastings. On the existence of homoclinic and periodic orbits in the FitzHugh-Nagumo equations. Quart. J. Math. Oxford, 2(27):123-134, 1976.

[18] J. Jalics, M. Krupa, and H.G. Rotstein. A novel canard-based mechanism for mixed-mode oscillations in a neuronal model. preprint, 2008.

[19] C. Jones and N. Kopell. Tracking invariant manifolds with differential forms in singularly perturbed systems. Journal of Differential Equations, pages 64-88, 1994.

[20] C. Jones, N. Kopell, and R. Langer. Construction of the FitzHugh-Nagumo pulse using differential forms. in: Multiple-Time-Scale Dynamical Systems, pages 101-113, 2001.

[21] Christopher K.R.T. Jones. Stability of the travelling wave solution of the FitzHugh-Nagumo system. Transactions of the American Mathematical Society, 286(2):431-469, 1984.

[22] M.T.M. Koper. Bifurcations of mixed-mode oscillations in a three-variable autonomous Van der PolDuffing model with a cross-shaped phase diagram. Physica D, 80:72-94, 1995.

[23] B. Krauskopf, H.M. Osinga, E.J. Doedel, M.E. Henderson, J. Guckenheimer, A. Vladimirsky, M. Dellnitz, and O. Junge. A survey of methods for computing (un)stable manifolds of vector fields. Int. J. Bifurcation and Chaos, 15(3):763-791, 2005.

[24] M. Krupa, N. Popovic, N. Kopell, and H.G. Rotstein. Mixed-mode oscillations in a three time-scale model for the dopaminergic neuron. Chaos, 18, 2008.

[25] Martin Krupa, Bjoern Sandstede, and Peter Szmolyan. Fast and slow waves in the FitzHugh-Nagumo equation. Journal of Differential Equations, 133:49-97, 1997.

[26] M. Krupa and P. Szmolyan. Relaxation oscillation and canard explosion. Journal of Differential Equations, 174:312-368, 2001.

[27] Yu.A. Kuznetsov. Elements of Applied Bifurcation Theory - $3^{\text {rd }}$ edition. Springer, 2004.

[28] A. Milik and P. Szmolyan. Multiple time scales and canards in a chemical oscillator. in: Multiple-TimeScale Dynamical Systems, Eds.: C.K.R.T. Jones and A.I. Khibnik, 2001.

[29] H.G. Rotstein, M. Wechselberger, and N. Kopell. Canard induced mixed-mode oscillations in a medial entorhinal cortex layer II stellate cell model. SIAM J. Applied Dynamical Systems, 7(4):1582-1611, 2008.

[30] J. Rubin and M. Wechselberger. The selection of mixed-mode oscillations in a Hodgin-Huxley model with multiple timescales. Chaos, 18, 2008. 
[31] L.F. Shampine and M.W. Reichelt. The MatLab ode suite. SIAM Jour. Sci. Comp., 18(1):1-22, 1997.

[32] L.P. Shilnikov A case of the existence of a denumerable set of periodic motions. Sov. Math. Dokl., 6:163-166, 1965.

[33] S.-K. Tin and N. Kopell and C.K.R.T. Jones. Invariant manifolds and singularly perturbed boundary value problems. SIAM J. Numer. Anal., 31(1):1558-1576, 1994. 